\title{
Energy Efficiency in the Low-SNR Regime under Queueing Constraints and Channel Uncertainty
}

\author{
Deli Qiao, Mustafa Cenk Gursoy, and Senem Velipasalar
}

\begin{abstract}
Energy efficiency of fixed-rate transmissions is studied in the presence of queueing constraints and channel uncertainty. It is assumed that neither the transmitter nor the receiver has channel side information prior to transmission. The channel coefficients are estimated at the receiver via minimum mean-square-error (MMSE) estimation with the aid of training symbols. It is further assumed that the system operates under statistical queueing constraints in the form of limitations on buffer violation probabilities. The optimal fraction of power allocated to training is identified. Spectral efficiency-bit energy tradeoff is analyzed in the low-power and wideband regimes by employing the effective capacity formulation. In particular, it is shown that the bit energy increases without bound in the low-power regime as the average power vanishes. A similar conclusion is reached in the wideband regime if the number of noninteracting subchannels grow without bound with increasing bandwidth. On the other hand, it is proven that if the number of resolvable independent paths and hence the number of noninteracting subchannels remain bounded as the available bandwidth increases, the bit energy diminishes to its minimum value in the wideband regime. For this case, expressions for the minimum bit energy and wideband slope are derived. Overall, energy costs of channel uncertainty and queueing constraints are identified, and the impact of multipath richness and sparsity is determined.
\end{abstract}

Index Terms: bit energy, channel estimation, effective capacity, energy efficiency, fading channels, fixed-rate transmission, imperfect channel knowledge, low-power regime, minimum bit energy, QoS constraints, spectral efficiency, wideband regime, wideband slope.

The authors are with the Department of Electrical Engineering, University of Nebraska-Lincoln, Lincoln, NE, 68588 (e-mails: dqiao726@ huskers.unl.edu, gursoy@engr.unl.edu, velipasa@engr.unl.edu).

This work was supported by the National Science Foundation under Grants CCF - 0546384 (CAREER) and CNS - 0834753. 


\section{INTRODUCTION}

In wireless communications, one of the main challenges in establishing reliable communications and providing quality of service guarantees is due to randomly varying channel conditions caused by mobility and changing environment. These time-varying channel conditions are often estimated in practical systems with the aid of pilot symbols albeit only imperfectly. Due to its practical significance, pilot-assisted wireless transmissions have been extensively studied in the literature. For instance, Hassibi and Hochwald in [1] obtained a capacity lower bound for pilot-assisted transmission in multiple-antenna fading channels, and identified the optimal training signal type, and its power and duration. In [2], the capacity and energyefficiency of training-based transmissions are investigated and the structure of the optimal input under peak power constraints is identified. In [3], an overview of pilot-assisted wireless transmission techniques and their performance analyses is provided.

In many wireless communication systems, satisfying certain quality of service (QoS) requirements is of paramount importance in providing acceptable performance and quality. For instance, in voice over IP (VoIP), interactive-video (e.g,. videoconferencing), and streaming-video applications in wireless systems, latency is a key QoS metric and should not exceed certain levels [28]. Recently, effective capacity is proposed in [11] as a metric that can be employed to measure the performance in the presence of statistical QoS limitations. Effective capacity formulation uses the large deviations theory and incorporates the statistical QoS constraints by capturing the rate of decay of the buffer occupancy probability for large queue lengths. Hence, effective capacity can be regarded as the maximum throughput of a system operating under limitations on the buffer violation probability. The analysis and application of effective capacity in various settings has attracted much interest recently (see e.g., [12]-[21] and references therein). For instance, Tang and Zhang in [14] considered the effective capacity when both the receiver and transmitter know the instantaneous channel gains, and derived the optimal power and rate adaptation technique that maximizes the system throughput under QoS constraints. Liu et al. in [18] considered fixed-rate transmission schemes and analyzed the effective capacity and related resource requirements for Markov wireless channel models. In this work, the continuous-time Gilbert-Elliott channel with ON and OFF states is adopted as the channel model while assuming the fading coefficients as zero-mean Gaussian distributed.

In addition to the above considerations, another important concern in wireless communications is energyefficient operation as mobile wireless systems can only be equipped with limited energy resources. To measure and compare the energy efficiencies of different systems and transmission schemes, one can choose 
as a metric the energy required to reliably send one bit of information. Information-theoretic studies show that energy-per-bit requirement is generally minimized, and hence the energy efficiency is maximized, if the system operates at low signal-to-noise ratio (SNR) levels and hence in the low-power or wideband regimes. Recently, Verdú in [8] determined the minimum bit energy required for reliable communication over a general class of channels by considering the Shannon capacity formulation, and studied of the spectral efficiency-bit energy tradeoff in the wideband regime. In [21] and [22], we incorporated the QoS limitations in the energy efficiency analysis by employing the effective capacity, rather than Shannon capacity, as the performance metric. We identified the bit energy requirements in the low-SNR regime. In particular, in [21], variable-rate/variable-power and variable-rate/fixed-power transmission schemes are studied assuming the availability of perfect channel side information (CSI) at both the transmitter and receiver or only at the receiver. In [22], the performance of fixed-rate/fixed-power transmissions is investigated when the receiver has perfect CSI while the transmitter has no such knowledge.

In this paper, as a major difference from the above-cited works, we jointly consider the three major challenges in wireless systems, namely communicating under channel uncertainty, providing QoS assurances, operating energy efficiently. We assume that the channel is not known by the transmitter and receiver prior to transmission, and is estimated imperfectly by the receiver through training. In our model, we incorporate statistical queueing constraints by employing the effective capacity formulation which provides the maximum throughput under limitations on buffer violation probabilities for large buffer sizes. Since the transmitter is assumed to not know the channel, fixed-rate transmission is considered. More specifically, the contributions of the paper are the following:

1) We provide a framework through which energy efficiency is measured in the presence of channel uncertainty and QoS limitations in the form of queueing constraints.

2) We obtain the optimal fraction of power that needs to be allocated to training in the presence of queueing constraints.

3) We determine the bit energy levels required for operation in the low-power and wideband regimes under channel uncertainty.

4) We identify the impact of rich and sparse multipath fading on the energy efficiency when the wideband channel is imperfectly known.

The rest of the paper is organized as follows. Section II introduces the system model and also delineates the training and data transmission phases, and the channel estimation method. In Section III, we briefly 
describe the notion of effective capacity and the spectral efficiency-bit energy tradeoff. Energy efficiency in the low-power regime is investigated in Section IV. In Section V, we analyze the energy efficiency in the wideband regime. Finally, Section VI provides conclusions.

\section{SySTEM MODEL}

We consider a point-to-point wireless link. Figure 1 illustrates the functional diagram of the system. It is assumed that the source generates data sequences which are divided into frames of duration $T$. These data frames are initially stored in the buffer before they are transmitted over the wireless channel. The discrete-time channel input-output relation in the $i^{\text {th }}$ symbol duration is given by

$$
y[i]=h[i] x[i]+n[i] \quad i=1,2, \ldots
$$

where $x[i]$ and $y[i]$ denote the complex-valued channel input and output, respectively. We assume that the bandwidth available in the system is $B$ and the channel input is subject to the following average energy

constraint: $\mathbb{E}\left\{|x[i]|^{2}\right\} \leq \bar{P} / B$ for all $i$. Since the bandwidth is $B$, symbol rate is assumed to be $B$ complex symbols per second, indicating that the average power of the system is limited by $\bar{P}$. Above in (1), $n[i]$ is a zero-mean, circularly symmetric, complex Gaussian random variable with variance $\mathbb{E}\left\{|n[i]|^{2}\right\}=N_{0}$, i.e., $n[i] \sim \mathcal{C N}\left(0, N_{0}\right)$. The additive Gaussian noise samples $\{n[i]\}$ are assumed to form an independent and identically distributed (i.i.d.) sequence. Finally, $h[i]$, which denotes the channel fading coefficient, is assumed to be a zero-mean Gaussian random variable with variance $E\left\{|h|^{2}\right\}=\gamma$. Therefore, the wireless channel is modeled as a Rayleigh fading channel. We further assume that the fading coefficients stay constant during the frame duration of $T$ seconds and have independent realizations for each frame. Hence, we basically consider a block-fading channel model. Finally, we assume that neither the transmitter nor the receiver has channel side information prior to transmission. While the transmitter remains unaware of the actual realizations of the fading coefficients throughout the transmission, the receiver attempts to learn them through training.

The system operates in two phases: training phase and data transmission phase. In the training phase, known pilot symbols are transmitted to enable the receiver to estimate the channel conditions, albeit imperfectly. We assume that minimum mean-square-error (MMSE) estimation is employed at the receiver to estimate the channel coefficient $h[i]$. Since the MMSE estimate depends only on the training energy and not on the training duration [1] and the fading coefficients are assumed to stay constant during the frame duration of $T$ seconds, it can be easily seen that transmission of a single pilot at every $T$ seconds is optimal. 
Note that in every frame duration of $T$ seconds, we have $T B$ symbols and the overall available energy is $\bar{P} T$. We now assume that each frame consists of a pilot symbol and $T B-1$ data symbols. The energies of the pilot and data symbols are

$$
\mathcal{E}_{t}=\rho \bar{P} T, \quad \text { and } \quad \mathcal{E}_{s}=\frac{(1-\rho) \bar{P} T}{T B-1}
$$

respectively, where $\rho$ is the fraction of total energy allocated to training. Note that the data symbol energy $\mathcal{E}_{s}$ is obtained by uniformly allocating the remaining energy among the data symbols.

In the training phase, the transmitter sends the pilot symbol $x_{t}=\sqrt{\mathcal{E}_{t}}=\sqrt{\rho \bar{P} T}$ and the receiver obtains

$$
y_{t}=h \sqrt{\mathcal{E}_{t}}+n
$$

Based on the received signal in this phase, the receiver obtains the MMSE estimate $\hat{h}=\mathbb{E}\left\{h \mid y_{t}\right\}$ which can be easily seen to be a circularly symmetric, complex, Gaussian random variable with mean zero and variance $\frac{\gamma^{2} \mathcal{E}_{t}}{\gamma \mathcal{E}_{t}+N_{0}}$, i.e., $\hat{h} \sim \mathcal{C N}\left(0, \frac{\gamma^{2} \mathcal{E}_{t}}{\gamma \mathcal{E}_{t}+N_{0}}\right)$ [2]. Now, the channel fading coefficient $h$ can be expressed as $h=\hat{h}+\tilde{h}$ where $\tilde{h}$ is the estimate error and $\tilde{h} \sim \mathcal{C N}\left(0, \frac{\gamma N_{0}}{\gamma \mathcal{E}_{t}+N_{0}}\right)$. Consequently, in the data transmission phase, the channel input-output relation becomes

$$
y[i]=\hat{h}[i] x[i]+\tilde{h}[i] x[i]+n[i] \quad i=1,2, \ldots, T B-1 .
$$

Since finding the capacity of the channel in (4) is a difficult task 1 , a capacity lower bound is generally obtained by considering the estimate error $\tilde{h}$ as another source of Gaussian noise and treating $\tilde{h}[i] x[i]+n[i]$ as Gaussian distributed noise uncorrelated from the input [1]. Now, the new noise variance is $\mathbb{E}\left\{|\tilde{h}[i] x[i]+n[i]|^{2}\right\}=$ $\sigma_{\tilde{h}}^{2} \mathcal{E}_{s}+N_{0}$ where $\sigma_{\tilde{h}}^{2}=\mathbb{E}\left\{|\tilde{h}|^{2}\right\}=\frac{\gamma N_{0}}{\gamma \mathcal{E}_{t}+N_{0}}$ is the variance of the estimate error. Under these assumptions, a lower bound on the instantaneous capacity is given by [1], [2]

$$
\begin{aligned}
C_{L} & =\frac{T B-1}{T} \log _{2}\left(1+\frac{\mathcal{E}_{s}}{\sigma_{\tilde{h}}^{2} \mathcal{E}_{s}+N_{0}}|\hat{h}|^{2}\right) \\
& =\frac{T B-1}{T} \log _{2}\left(1+\mathrm{SNR}_{\mathrm{eff}}|w|^{2}\right) \text { bits/s }
\end{aligned}
$$

\footnotetext{
${ }^{1}$ In [2], the capacity of training-based transmissions under input peak power constraints is shown to be achieved by an SNR-dependent, discrete distribution with a finite number of mass points. In such cases, no closed-form expression for the capacity exists, and capacity values need to be obtained through numerical computations.
} 
where the effective SNR is

$$
\mathrm{SNR}_{\mathrm{eff}}=\frac{\mathcal{E}_{s} \sigma_{\hat{h}}^{2}}{\sigma_{\tilde{h}}^{2} \mathcal{E}_{s}+N_{0}},
$$

and $\sigma_{\hat{h}}^{2}=\mathbb{E}\left\{|\hat{h}|^{2}\right\}=\frac{\gamma^{2} \mathcal{E}_{t}}{\gamma \mathcal{E}_{t}+N_{0}}$ is the variance of the estimate $\hat{h}$. Note that the expression in (6) is obtained by defining $\hat{h}=\sigma_{\hat{h}} w$ where $w$ is a standard complex Gaussian random variable with zero mean and unit variance, i.e., $w \sim \mathcal{C N}(0,1)$.

Since Gaussian is the worst uncorrelated noise [1], the above-mentioned assumptions lead to a pessimistic model and the rate expression in (6) is a lower bound to the capacity of the true channel (4). On the other hand, $C_{L}$ is a good measure of the rates achieved in communication systems that operate as if the channel estimate were perfect (i.e., in systems where Gaussian codebooks designed for known channels are used, and scaled nearest neighbor decoding is employed at the receiver) [4]. Henceforth, we base our analysis on $C_{L}$ to understand the impact of the imperfect channel estimate.

Since the transmitter is unaware of the channel conditions, it is assumed that information is transmitted at a fixed rate of $r$ bits/s. When $r<C_{L}$, the channel is considered to be in the ON state and reliable communication is achieved at this rate. Note that under the block-fading assumption, the channel stays in the ON state for $T$ seconds and the number of bits transmitted in this duration is $r T$. If, on the other hand, $r \geq C_{L}$, we assume that outage occurs. In this case, channel is in the OFF state during the frame duration and reliable communication at the rate of $r$ bits/s cannot be attained. Hence, effective data rate is zero and information has to be resent. Fig. 2 depicts the two-state transmission model together with the transition probabilities. Under the assumption of independent fading realizations in different blocks of duration $T$, it can be easily seen that the transition probabilities are given by

$$
\begin{aligned}
& p_{11}=p_{21}=P\left\{r \geq C_{L}\right\}=P\left\{|w|^{2} \leq \alpha\right\} \\
& p_{22}=p_{12}=P\left\{r<C_{L}\right\}=P\left\{|w|^{2}>\alpha\right\}
\end{aligned}
$$

where

$$
\alpha=\frac{2^{\frac{r T}{T B-1}}-1}{\mathrm{SNR}_{\mathrm{eff}}},
$$

and $|w|^{2}$ is an exponential random variable with mean 1, and hence, $P\left\{|w|^{2}>\alpha\right\}=e^{-\alpha}$. 


\section{PRELIMINARIES}

\section{A. Effective Capacity}

In [11], Wu and Negi defined the effective capacity as the maximum constant arrival rate that a given service process can support in order to guarantee a statistical QoS requirement specified by the QoS exponent $\theta$ 2. If we define $Q$ as the stationary queue length, then $\theta$ is the decay rate of the tail distribution of the queue length $Q$ :

$$
\lim _{q \rightarrow \infty} \frac{\log P(Q \geq q)}{q}=-\theta
$$

Therefore, for large $q_{\max }$, we have the following approximation for the buffer violation probability: $P(Q \geq$ $\left.q_{\max }\right) \approx e^{-\theta q_{\max }}$. Hence, while larger $\theta$ corresponds to more strict QoS constraints, smaller $\theta$ implies looser QoS guarantees. Similarly, if $D$ denotes the steady-state delay experienced in the buffer, then $P(D \geq$ $\left.d_{\max }\right) \approx e^{-\theta \delta d_{\max }}$ for large $d_{\max }$, where $\delta$ is determined by the arrival and service processes [17]. Therefore, effective capacity formulation provides the maximum constant arrival rates that can be supported by the time-varying wireless channel under the queue length constraint $P\left(Q \geq q_{\max }\right) \leq e^{-\theta q_{\max }}$ for large $q_{\max }$ or the delay constraint $P\left(D \geq d_{\max }\right) \leq e^{-\theta \delta d_{\max }}$ for large $d_{\max }$. Since the average arrival rate is equal to the average departure rate when the queue is in steady-state [25], effective capacity can also be seen as the maximum throughput in the presence of such constraints.

The effective capacity is given by ([11], [23], [24])

$$
-\frac{\Lambda(-\theta)}{\theta}=-\lim _{t \rightarrow \infty} \frac{1}{\theta t} \log _{e} \mathbb{E}\left\{e^{-\theta S[t]}\right\}
$$

where $S[t]=\sum_{i=1}^{t} R[i]$ is the time-accumulated service process and $\{R[i], i=1,2, \ldots\}$ denote the discretetime stationary and ergodic stochastic service process. Note that in the model we consider, $R[i]=r T$ or 0 depending on the channel state being ON or OFF. In [24], it is shown that for such an ON-OFF model, we have

$$
\frac{\Lambda(\theta)}{\theta}=\frac{1}{\theta} \log _{e}\left(\frac{1}{2}\left(p_{11}+p_{22} e^{\theta T r}+\sqrt{\left(p_{11}+p_{22} e^{\theta T r}\right)^{2}+4\left(p_{11}+p_{22}-1\right) e^{\theta T r}}\right)\right) .
$$

Note that $p_{11}+p_{22}=1$ in our model. Then, for a given QoS delay constraint $\theta$, the effective capacity normalized by the frame duration $T$ and bandwidth $B$, or equivalently spectral efficiency in bits/s/Hz,

\footnotetext{
${ }^{2}$ For time-varying arrival rates, effective capacity specifies the effective bandwidth of the arrival process that can be supported by the channel.
} 
becomes

$$
\begin{aligned}
\mathrm{R}_{E}(\mathrm{SNR}, \theta) & =\max _{\substack{r \geq 0 \\
0 \leq \rho \leq 1}}-\frac{1}{T B} \frac{\Lambda(-\theta)}{\theta} \quad \text { bits/s/Hz } \\
& =\max _{\substack{r \geq 0 \\
0 \leq \rho \leq 1}}-\frac{1}{\theta T B} \log _{e}\left(p_{11}+p_{22} e^{-\theta T r}\right) \\
& =\max _{\substack{r \geq 0 \\
0 \leq \rho \leq 1}}-\frac{1}{\theta T B} \log _{e}\left(1-P\left(|w|^{2}>\alpha\right)\left(1-e^{-\theta T r}\right)\right) \\
& =-\frac{1}{\theta T B} \log _{e}\left(1-P\left(|w|^{2}>\alpha_{\mathrm{opt}}\right)\left(1-e^{-\theta T r_{\mathrm{opt}}}\right)\right) .
\end{aligned}
$$

Note that $\mathrm{R}_{E}$ is obtained by optimizing both the fixed transmission rate $r$ and the fraction of power allocated to training, $\rho$. In the optimization result (17), $r_{\mathrm{opt}}$ and $\alpha_{\mathrm{opt}}$ are the optimal values of $r$ and $\alpha$, respectively. $r_{\text {opt }}$ can be found by solving

$$
\frac{2^{\frac{T r}{T B-1}} T \log _{e} 2}{(T B-1) \mathrm{SNR}_{\mathrm{eff}}}\left(1-e^{-\theta T r}\right)-\theta T e^{-\theta T r}=0
$$

where the left-hand side of (18) is the first derivative of the objective function in (16) with respect to $r$.

It can easily be seen that

$$
\mathrm{R}_{E}(\mathrm{SNR}, 0)=\lim _{\theta \rightarrow 0} \mathrm{R}_{E}(\mathrm{SNR}, \theta)=\max _{r \geq 0} \frac{r}{B} P\left\{|w|^{2}>\frac{2^{\frac{r T}{T B-1}}-1}{\mathrm{SNR}_{\mathrm{eff}}}\right\} .
$$

Hence, as the QoS requirements relax, the maximum constant arrival rate approaches the average transmission rate. On the other hand, for $\theta>0, \mathrm{R}_{E}<\frac{1}{B} \max _{r \geq 0} r P\left(|w|^{2}>\alpha\right)$ in order to avoid violations of buffer constraints.

\section{B. Spectral Efficiency-Bit Energy Tradeoff in the Low-SNR regime}

In this paper, we focus on the energy efficiency of wireless transmissions under the aforementioned statistical queueing constraints. Since energy efficient operation generally requires operation at low-SNR levels, our analysis throughout the paper is carried out in the low-SNR regime. In this regime, the tradeoff between the normalized effective capacity (i.e, spectral efficiency) $\mathrm{R}_{E}$ and bit energy $\frac{E_{b}}{N_{0}}=\frac{\mathrm{SNR}}{\mathrm{R}_{E}(\mathrm{SNR})}$ is a key tradeoff in understanding the energy efficiency, and is characterized by the bit energy at zero spectral efficiency and wideband slope provided, respectively, by

$$
\left.\frac{E_{b}}{N_{0}}\right|_{\mathrm{R}_{E}=0}=\frac{1}{\dot{\mathrm{R}}_{E}(0)} \text { and } \mathcal{S}_{0}=-\frac{2\left(\dot{\mathrm{R}_{E}}(0)\right)^{2}}{\mathrm{R}_{E}(0)} \log _{e} 2
$$


where $\dot{\mathrm{R}}_{E}(0)$ and $\ddot{\mathrm{R}}_{E}(0)$ are the first and second derivatives with respect to SNR, respectively, of the function $\mathrm{R}_{E}$ (SNR) at zero SNR [8]. $\left.\frac{E_{b}}{N_{0}}\right|_{\mathrm{R}_{E}=0}$ specifies the bit energy required as SNR vanishes or equivalently as $\mathrm{R}_{E} \rightarrow 0$, while $\mathcal{S}_{0}$ provides the slope of the spectral efficiency curve at $\left.\frac{E_{b}}{N_{0}}\right|_{\mathrm{R}_{E}=0}$. Therefore, $\left.\frac{E_{b}}{N_{0}}\right|_{\mathrm{R}_{E}=0}$ and $\mathcal{S}_{0}$ provide a linear approximation of the spectral-efficiency vs. bit energy curve at small SNR levels. We also note that in certain cases, the bit energy required for reliable communications diminishes with decreasing spectral efficiency, and we have $\left.\frac{E_{b}}{N_{0}}\right|_{\mathrm{R}_{E}=0}=\frac{E_{b}}{N_{0} \min }$.

\section{ENERGy EFFICIENCY In THE LOW-POWER REGIME}

In this section, we analyze the spectral-efficiency vs. bit energy tradeoff in the low power regime in which the the average power of the system, $\bar{P}$, is small. However, before the low-power analysis, we first obtain the following result on the optimal value of $\rho$. Note that this result is general and applies at all SNR levels.

Theorem 1: At a given SNR level, the optimal fraction of power $\rho_{\mathrm{opt}}$ that solves (16) does not depend on the QoS exponent $\theta$ and the transmission rate $r$, and is given by

$$
\rho_{\mathrm{opt}}=\sqrt{\eta(\eta+1)}-\eta
$$

where

$$
\eta=\frac{\gamma T B \mathrm{SNR}+T B-1}{\gamma T B(T B-2) \mathrm{SNR}} \quad \text { and } \quad \mathrm{SNR}=\frac{\bar{P}}{N_{0} B} .
$$

Proof: From (16) and the definition of $\alpha$ in (10), we can easily see that for fixed $r$, the only term in (16) that depends on $\rho$ is $\alpha$. Moreover, $\alpha$ has this dependency through $\mathrm{SNR}_{\mathrm{eff}}$. Therefore, $\rho_{\mathrm{opt}}$ that maximizes the objective function in (16) can be found by minimizing $\alpha$, or equivalently maximizing SNR eff $_{\text {. Substituting }}$ the definitions in (2) and the expressions for $\sigma_{\hat{h}}^{2}$ and $\sigma_{\tilde{h}}^{2}$ into (7), we have

$$
\mathrm{SNR}_{\mathrm{eff}}=\frac{\mathcal{E}_{s} \sigma_{\hat{h}}^{2}}{\sigma_{\tilde{h}}^{2} \mathcal{E}_{s}+N_{0}}=\frac{\rho(1-\rho) \gamma^{2} T^{2} B^{2} \mathrm{SNR}^{2}}{\rho \gamma T B(T B-2) \mathrm{SNR}+\gamma T B \mathrm{SNR}+T B-1}
$$

where SNR $=\frac{\bar{P}}{N_{0} B}$. Evaluating the derivative of $\mathrm{SNR}_{\mathrm{eff}}$ with respect to $\rho$ and making it equal to zero leads to the expression in (21). Clearly, $\rho_{\mathrm{opt}}$ is independent of $\theta$ and $r$.

Above, we have implicitly assumed that the maximization is performed with respect to first $\rho$ and then $r$. However, the result will not alter if the order of the maximization is changed. Note that the objective 
function in (16)

$$
g\left(\mathrm{SNR}_{\mathrm{eff}}, r\right)=-\frac{1}{\theta T B} \log _{e}\left(1-P\left(|w|^{2}>\frac{2^{\frac{r T}{T B-1}}-1}{\mathrm{SNR}_{\mathrm{eff}}}\right)\left(1-e^{-\theta T r}\right)\right)
$$

is a monotonically increasing function of $\mathrm{SNR}_{\mathrm{eff}}$ for all $r$. It can be easily verified that maximization does not affect the monotonicity of $g$, and hence $\max _{r \geq 0} g\left(\mathrm{SNR}_{\mathrm{eff}}, r\right)$ is still a monotonically increasing function of $\mathrm{SNR}_{\mathrm{eff}}$. Therefore, in the outer maximization with respect to $\rho$, the choice of $\rho$ that maximizes SNR $\mathrm{eff}_{\text {will }}$ also maximize $\max _{r \geq 0} g\left(\mathrm{SNR}_{\mathrm{eff}}, r\right)$, and the optimal value of $\rho$ is again given by (21).

Fig. 3 plots $\rho_{\text {opt }}$, the optimal fraction of power allocated to training, as a function of SNR for different values of $\theta$ when $B=10^{7} \mathrm{~Hz}$. As predicted, $\rho_{\mathrm{opt}}$ is the same for all $\theta$. Note that as SNR $\rightarrow 0$, we have $\eta \rightarrow \infty$ and $\rho_{\mathrm{opt}} \rightarrow 1 / 2$, which is also observed in the figure. We further observe in Fig. 3 that $\rho_{\mathrm{opt}}$ decreases with increasing SNR. Moreover, as SNR $\rightarrow \infty$, we can find that $\eta \rightarrow \frac{1}{T B-2}$ and hence $\rho_{\text {opt }} \rightarrow \sqrt{\frac{1}{T B-2}\left(\frac{1}{T B-2}+1\right)}-$ $\frac{1}{T B-2}$. In the figure, we assume $T=2 \mathrm{~ms}$, and therefore $T B=2 \times 10^{4}$ and $\rho_{\mathrm{opt}} \rightarrow 0.007$.

With the optimal value of $\rho$ given in Theorem 1, we can now express the normalized effective capacity as

$$
\begin{aligned}
\mathrm{R}_{E}(\mathrm{SNR}, \theta) & =\max _{r \geq 0}-\frac{1}{\theta T B} \log _{e}\left(1-P\left(|w|^{2}>\frac{2^{\frac{r T}{T B-1}}-1}{\mathrm{SNR}_{\mathrm{eff}, \mathrm{opt}}}\right)\left(1-e^{-\theta T r}\right)\right) \\
& =-\frac{1}{\theta T B} \log _{e}\left(1-P\left(|w|^{2}>\frac{2^{\frac{r_{\mathrm{opt} T} T}{T B-1}}-1}{\mathrm{SNR}_{\mathrm{eff}, \mathrm{opt}}}\right)\left(1-e^{-\theta T r_{\mathrm{opt}}}\right)\right)
\end{aligned}
$$

where $r_{\mathrm{opt}}$ is the optimal value of $r$ that solves (25), and

$$
\mathrm{SNR}_{\mathrm{eff}, \mathrm{opt}}=\frac{\phi(\mathrm{SNR}) \mathrm{SNR}^{2}}{\psi(\mathrm{SNR}) \mathrm{SNR}+T B-1}
$$

and

$$
\phi(\mathrm{SNR})=\rho_{\mathrm{opt}}\left(1-\rho_{\mathrm{opt}}\right) \gamma^{2} T^{2} B^{2}, \text { and } \psi(\mathrm{SNR})=\left(1+(T B-2) \rho_{\mathrm{opt}}\right) \gamma T B
$$

With these notations, we obtain the following result that shows us that operation at very low power levels is extremely energy inefficient and should be avoided.

Theorem 2: In the presence of channel uncertainty, the bit energy for all $\theta \geq 0$ increases without bound as the average power $\bar{P}$ and hence SNR vanishes, i.e.,

$$
\left.\frac{E_{b}}{N_{0}}\right|_{\mathrm{R}_{E}=0}=\lim _{\mathrm{SNR} \rightarrow 0} \frac{E_{b}}{N_{0}}=\lim _{\mathrm{SNR} \rightarrow 0} \frac{\mathrm{SNR}}{\mathrm{R}_{E}(\mathrm{SNR})}=\frac{1}{\dot{\mathrm{R}_{E}(0)}}=\infty
$$


Proof: Recall that $|w|^{2}$ is an exponetial random variable with mean 1 and hence $P\left\{|w|^{2}>\alpha\right\}=e^{-\alpha}$. Moreover, note that as SNR $\rightarrow 0$, transmission rates also approach zero and therefore we have $r_{\mathrm{opt}} \rightarrow 0$. Using these facts, it can be shown that the derivative of $\mathrm{R}_{E}$ in (26) with respect to $\mathrm{SNR}$ at $\mathrm{SNR}=0$ is

$$
\dot{\mathrm{R}}_{E}(0)=\lim _{\mathrm{SNR} \rightarrow 0} \frac{1}{B} e^{-\alpha_{\mathrm{opt}}} \dot{r}_{\mathrm{opt}} e^{-\theta T r_{\mathrm{opt}}}-\frac{1}{\theta T B} \dot{\alpha}_{\mathrm{opt}} e^{-\alpha_{\mathrm{opt}}}\left(1-e^{-\theta T r_{\mathrm{opt}}}\right)
$$

where $\dot{r}_{\mathrm{opt}}$ and $\dot{\alpha}_{\mathrm{opt}}$ are the derivatives of $r_{\mathrm{opt}}$ and $\alpha_{\mathrm{opt}}$, respectively, with respect to SNR, and $\alpha_{\mathrm{opt}}=\frac{2 \frac{r_{\mathrm{opp} T} T}{\mathrm{~TB}-1}-1}{\mathrm{SNR}_{\mathrm{eff}, \mathrm{opt}}}$.

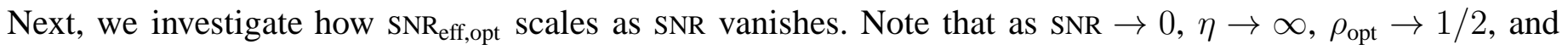
hence $\phi(\mathrm{SNR}) \rightarrow 1 / 4 \gamma^{2} T^{2} B^{2}$. Then, we have

$$
\mathrm{SNR}_{\mathrm{eff}, \mathrm{opt}}=\frac{\gamma^{2} T^{2} B^{2}}{4(T B-1)} \mathrm{SNR}^{2}+o\left(\mathrm{SNR}^{2}\right) .
$$

Therefore, $\mathrm{SNR}_{\mathrm{eff}, \mathrm{opt}}$ decreases as $\mathrm{SNR}^{2}$ as SNR diminishes to zero. Now, we consider the behavior of $r_{\mathrm{opt}}$ at low SNRs. If $r_{\mathrm{opt}}$ diminishes slower than $\mathrm{SNR}^{2}$ (for instance, if $r_{\mathrm{opt}}$ decreases as $\mathrm{SNR}^{a}$ where $0<a<2$ ), then it can be verified that $\alpha_{\text {opt }} \rightarrow \infty$ as SNR $\rightarrow 0$ from which we can immediately see that $\dot{\mathrm{R}}_{E}(0)=0$ due to exponentially decreasing term $e^{-\alpha_{\mathrm{opt}}}$. On the other hand, if $r_{\mathrm{opt}}$ reduces to zero faster than or as $\mathrm{SNR}^{2}$ (e.g., as $\mathrm{SNR}^{a}$ where $\left.a \geq 2\right), \alpha_{\mathrm{opt}}$ approaches a finite value. However in this case, we can show that $\dot{r}_{\mathrm{opt}} \rightarrow 0$ and $\dot{\alpha}_{\text {opt }}\left(1-e^{-\theta T r_{\text {opt }}}\right) \rightarrow 0$ as SNR $\rightarrow 0$, leading again the conclusion that $\dot{\mathrm{R}}_{E}(0)=0$.

Remark: Theorem 2 shows that $\left.\frac{E_{b}}{N_{0}}\right|_{\mathrm{R}_{E}=0}=\infty$ for any $\theta \geq 0$. Hence, as noted before, operation at very low power levels is extremely energy inefficient. One reason for this behavior is that although channel estimation at very low power levels does not provide reliable estimates, the receiver regards this estimate as perfect. Hence, in the low-power regime, we have both diminishing power and deteriorating channel estimate, which affect the performance adversely. The result of Theorem 2 also indicates that the minimum bit energy, which can be identified numerically, is achieved at a non-zero power level. In the numerical results, we will observe that both the minimum required bit energy and the other bit energy values required at a given level of spectral efficiency increase as the QoS constraints become more stringent.

Fig. 4 plots the spectral efficiency vs. bit energy for $\theta=\{1,0.1,0.01,0.001\}$ when $B=10^{5} \mathrm{~Hz}$ in Rayleigh channel with $\mathbb{E}\left\{|h|^{2}\right\}=\gamma=1$. We notice that as spectral efficiency $\mathrm{R}_{E}$ decreases, the bit energy $\frac{E_{b}}{N_{0}}$ initially decreases. However, as predicted by the result of Theorem 2 , the bit energy achieves its minimum value at a certain nonzero spectral efficiency below which $\frac{E_{b}}{N_{0}}$ starts increasing without bound. Hence, operation below the spectral efficiency or SNR level at which $\frac{E_{b}}{N_{0}}$ min is attained should be avoided. We also note in Fig. 4 that the bit energy requirements in general and the minimum bit energy in particular increases with 
increasing $\theta$ value, indicating the increased energy costs as the QoS limitations become more stringent. In Fig. 5, we plot $\frac{E_{b}}{N_{0}}$ as a function of SNR for different bandwidth levels assuming $\theta=0.01$. We again observe that the minimum bit energy is attained at a nonzero SNR value below which $\frac{E_{b}}{N_{0}}$ requirements start increasing. Furthermore, we see that as the bandwidth increases, the minimum bit energy tends to decrease and is achieved at a lower SNR level. Finally, we plot in Fig. 6 the minimum bit energy as a function of the bandwidth, $B$. We note that increasing $B$ generally decreases $\frac{E_{b}}{N_{0}}$ min value. However, there is diminishing returns as $B$ gets larger. Analysis in the wideband regime in the following section will provide more insight into the impact of large bandwidth.

\section{ENERgy EFFICIENCY IN THE Wideband REgime}

In this section, we consider the wideband regime in which the bandwidth is large. We assume that the average power $\bar{P}$ is kept constant. Note that as the bandwidth $B$ increases, SNR $=\frac{\bar{P}}{N_{0} B}$ approaches zero and we operate in the low-SNR regime.

In Section I, we have described a flat fading channel model. However, flat fading assumption will not hold in the wideband regime as the bandwidth $B$ increases without bound. On the other hand, if we decompose the wideband channel into $N$ parallel subchannels, and suppose that each subchannel has a bandwidth that is equal to the coherence bandwidth, $B_{c}$, then we can assume that independent flat-fading is experienced in each subchannel. Note that we have $B=N B_{c}$. Similar to (1), the input-output relation in the $k^{\text {th }}$ subchannel can be written as

$$
y_{k}[i]=h_{k}[i] x_{k}[i]+n_{k}[i] \quad i=1,2, \ldots \quad \text { and } \quad k=1,2, \ldots, N
$$

The fading coefficients $\left\{h_{k}\right\}_{k=1}^{N}$ in different subchannels are assumed to be independent zero-mean Gaussian distributed with variances $\mathbb{E}\left\{\left|h_{k}\right|^{2}\right\}=\gamma_{k}$. The signal-to-noise ratio in the $k^{\text {th }}$ subchannel is $\operatorname{SNR}_{k}=\frac{\bar{P}_{k}}{N_{0} B_{c}}$ where $\bar{P}_{k}$ denotes the power allocated to the $k^{\text {th }}$ subchannel and we have $\sum_{k=1}^{N} \bar{P}_{k}=\bar{P} 3$. Over each subchannel, the same transmission strategy as described in Section $\amalg$ is employed. Therefore, the transmitter, not knowing the fading coefficients of the subchannels, sends the data over each subchannel at the fixed rate of $r$. Now, we can find that $C_{L, k}$ for each subchannel is given by $\frac{T B_{c}-1}{T} \log _{2}\left(1+\mathrm{SNR}_{\mathrm{eff}, k}|w|^{2}\right)$ bits/s, in which

$$
\mathrm{SNR}_{\mathrm{eff}, k}=\frac{\mathcal{E}_{s, k} \sigma_{\hat{h_{k}}}^{2}}{\sigma_{\tilde{h_{k}}}^{2} \mathcal{E}_{s, k}+N_{0}}
$$

\footnotetext{
${ }^{3}$ While not equipped with the knowledge of the instantaneous values of the fading coefficients, the transmitter is assumed to know the statistics of the fading coefficients, and possibly allocate different power levels to different subchannels with this knowledge.
} 
where $\mathcal{E}_{s, k}=\frac{\left(1-\rho_{k}\right) T \bar{P}_{k}}{T B_{c}-1}, \mathcal{E}_{t, k}=\rho_{k} T \bar{P}_{k}, \sigma_{\tilde{h_{k}}}^{2}=\frac{\gamma_{k} N_{0}}{\gamma_{k} \mathcal{E}_{t, k}+N_{0}}$ and $\sigma_{\hat{h_{k}}}^{2}=\frac{\gamma_{k}^{2} \mathcal{E}_{t, k}}{\gamma_{k} \mathcal{E}_{t, k}+N_{0}}$. Similarly as before, if $r<C_{L, k}$, then transmission over the $k^{\text {th }}$ subchannel is successful. Otherwise, retransmission is required. Hence, we have an ON-OFF state model for each subchannel. On the other hand, for the transmission over $N$ subchannels, we have a state-transition model with $N+1$ states because we have overall the following $N+1$ possible total transmission rates: $\{0, r T, 2 r T, \ldots, N r T\}$. For instance, if all $N$ subchannels are in the OFF state simultaneously, the total rate is zero. If $j$ out of $N$ subchannels are in the ON state, then the rate is $j r T$. We note that such a decomposition strategy is also employed in [22] where the receiver is assumed to have perfect channel information. Although similar, this strategy is also discussed here for the sake of completeness.

Now, assume that the states are enumerated in the increasing of order of the total transmission rates supported by them. Hence, in state $j \in\{1, \ldots, N+1\}$, the transmission rate is $(j-1) r T$. The transition probability from state $i \in\{1, \ldots, N+1\}$ to state $j \in\{1, \ldots, N+1\}$ is given by

$$
\begin{aligned}
p_{i j}=p_{j} & =P\{(j-1) \text { subchannels out of } N \text { subchannels are in the ON state }\} \\
& =\sum_{\mathcal{I}_{j-1} \subset\{1, \ldots, N\}}\left(\prod_{k \in \mathcal{I}_{j-1}} P\left\{|w|^{2}>\alpha_{k}\right\} \prod_{k \in \mathcal{I}_{j-1}^{c}}\left(1-P\left\{|w|^{2}>\alpha_{k}\right\}\right)\right)
\end{aligned}
$$

where $\mathcal{I}_{j-1}$ denotes a subset of the index set $\{1, \ldots, N\}$ with $j-1$ elements. The summation in (35) is over all such subsets. Moreover, in (35), $\mathcal{I}_{j-1}^{c}$ denotes the complement of the set $\mathcal{I}_{j-1}$, and $\alpha_{k}=\frac{2^{\frac{r T}{T_{c}-1}}-1}{\mathrm{SNR}_{\mathrm{eff}, k}}$. Note in the above formulation that the transition probabilities, $p_{i, j}$, do not depend on the initial state $i$ due to the block-fading assumption. If, in addition to being independent, the fading coefficients $h_{k}$ in different subchannels are identically distributed (i.e., the variances $\left\{\gamma_{k}\right\}_{k=1}^{N}$ are the same) and also if the total power is uniformly distributed over the subchannels and the fraction of energy, $\rho_{k}$, allocated to training in each subchannel is the same, then $p_{i, j}$ in (35) simplifies and becomes a binomial probability:

$$
p_{i, j}=p_{j}=\left(\begin{array}{c}
N \\
j-1
\end{array}\right)\left(P\left\{|w|^{2}>\alpha\right\}\right)^{j-1}\left(1-P\left\{|w|^{2}>\alpha\right\}\right)^{N-j+1}
$$

Note that with equal power allocation, we have $\bar{P}_{k}=\frac{\bar{P}}{N}$ and therefore $\mathrm{SNR}_{k}=\frac{\bar{P}_{k}}{N_{0} B_{c}}=\frac{\bar{P} / N}{N_{0} B / N}=\frac{\bar{P}}{N_{0} B}=\operatorname{SNR}$ which is equal to the original SNR used in (22). Since $\left\{\mathrm{SNR}_{\mathrm{eff}, k}\right\}_{k=1}^{N}$ are also equal due to having equal $\rho_{k}$ 's, we have the same $\alpha=\frac{2 \frac{r T}{T B_{c}-1}-1}{\mathrm{SNR}_{\mathrm{eff}}}$ for each subchannel.

The effective capacity of this wideband channel model with $\mathrm{N}$ noninteracting subchannels is given by the 
following result.

Theorem 3: For the wideband channel with $N$ parallel noninteracting subchannels each with bandwidth $B_{c}$ and independent flat fading, the normalized effective capacity in bits/s/ $\mathrm{Hz}$ is given by

$$
\mathrm{R}_{E}(\mathrm{SNR}, \theta)=\max _{\substack{r \geq 0 \\ \bar{P}_{k} \geq 0 . \rho_{k} \leq \bar{P}_{k} \leq \bar{P} \\ 0 \leq \rho_{k} \leq 1}}\left\{-\frac{1}{\theta T B} \log _{e}\left(\sum_{j=1}^{N+1} p_{j} e^{-\theta(j-1) r T}\right)\right\}
$$

where $p_{j}$ is given in (35). If $\left\{h_{k}\right\}_{k=1}^{N}$ are identically distributed Gaussian random variables with zero mean and variance $\gamma$ and the data and training energies are uniformly allocated over the subchannels, then the normalized effective capacity expression simplifies to

$$
\mathrm{R}_{E}(\mathrm{SNR}, \theta)=\max _{\substack{r \geq 0 \\ 0 \leq \rho \leq 1}}\left\{-\frac{1}{\theta T B_{c}} \log _{e}\left(1-P\left\{|w|^{2}>\alpha\right\}\left(1-e^{-\theta T r}\right)\right)\right\}
$$

where $\alpha=\frac{2^{\frac{r T}{T B_{c}-1}}-1}{\mathrm{SNR}_{\text {eff }}}$ and $\mathrm{SNR}_{\text {eff }}=\frac{\rho(1-\rho) \gamma^{2} T^{2} B_{c}^{2} \mathrm{SNR}^{2}}{\rho \gamma T B_{c}\left(T B_{c}-2\right) \mathrm{SNR}+\gamma T B_{c} \mathrm{SNR}+T B_{c}-1}$, in which $\mathrm{SNR}=\frac{\bar{P}}{N_{0} B}=\frac{\bar{P}}{N N_{0} B_{c}}$.

Proof: See [22, Appendix A].

Remark: Theorem 3 shows that if the fading coefficients in different subchannels are i.i.d. and the data and training energies are uniformly allocated over the subchannels, then the effective capacity of a wideband channel has an expression similar to that in (16), which provides the effective capacity of a single channel experiencing flat fading. The only difference between (16) and (38) is that $B$ is replaced in (38) by $B_{c}$, which is the bandwidth of each subchannel.

As mentioned before, we in this section consider the wideband regime in which the overall bandwidth of the system, $B$, is large. Additionally, we henceforth limit our analysis to the case in which the effective capacity is given by (38) because optimization over the power allocation schemes and obtaining closed-form expressions are in general difficult tasks in the wideband regime in which the number of subchannels is potentially high. Under these assumptions, we investigate two scenarios:

1) Rich multipath fading: In this case, we assume that the number of independent resolvable paths increases linearly with the bandwidth. This in turn implies that as the bandwidth $B$ increases, the number of noninteracting subchannels $N$ increases while $B_{c}$ stays fixed.

2) Sparse multipath fading: In this case, we assume that the number of independent resolvable paths increases at most sublinearly with the bandwidth. This assumption implies the coherence bandwidth $B_{c}=\frac{B}{N}$ increases with increasing bandwidth $B$ [5], [6]. We can identify two subcases: 
a) If the number of resolvable paths remains bounded in the wideband regime (as considered for instance in [7]), then $N$ remains bounded while $B_{c}$ increases linearly with $B$.

b) If the number of resolvable paths increases but only sublinearly with $B$, then both $N$ and $B_{c}$ grow without bound with $B$.

We first consider scenario (1) where rich multipath fading is assumed. In this case, as $B$ increases, the signalto-noise ratio SNR $=\frac{\bar{P}}{N_{0} B}=\frac{\bar{P}}{N N_{0} B_{c}}$ approaches zero while $B_{c}$ stays fixed. From these facts and the similarity of the formulations in (16) and (38), we immediately conclude that the wideband regime analysis of the rich multipath case is the same as the low-power regime analysis conducted in Section IV] Therefore, as $B \rightarrow \infty$ in the rich multipath fading scenario, we have $\left.\frac{E_{b}}{N_{0}}\right|_{\mathrm{R}_{E}=0}=\lim _{\mathrm{SNR} \rightarrow 0} \frac{E_{b}}{N_{0}}=\infty$ for all $\theta \geq 0$. Note that we have high diversity in rich multipath fading as the number of noninteracting subchannels increase linearly with bandwidth. On the other hand, since independent fading coefficients are only imperfectly known and moreover the receiver's ability to estimate the subchannels diminishes with decreasing SNR, we have high uncertainty as well. Hence, uncertainty becomes the more dominant factor and extreme energy-inefficiency is experienced in the limit as $B \rightarrow \infty$.

Next, we analyze the performance in the scenario of sparse multipath fading. We note that the authors in [5] and [6], motivated by the recent measurement studies in the ultrawideband regime, considered sparse multipath fading channels and analyzed the performance under channel uncertainty, employing the Shannon capacity formulation as the performance metric. We in this paper consider channel uncertainty and queueing constraints jointly and use the effective capacity to identify the performance. We first consider scenario (2a) where the the number of subchannels $N$ remains bounded and the degrees of freedom are limited. The following result provides the expressions for the bit energy at zero spectral efficiency and the wideband slope, and characterize the spectral efficiency-bit energy tradeoff in the wideband regime when $N$ is fixed and $B_{c}$ grows linearly with $B$. It is shown that the bit energy required at zero spectral efficiency is indeed the minimum bit energy.

Theorem 4: For sparse multipath fading channel with bounded number of independent resolvable paths, the minimum bit energy and wideband slope in the wideband regime are given by

$$
\begin{gathered}
\frac{E_{b}}{N_{0 \text { min }}}=\frac{-\delta \log _{e} 2}{\log _{e} \xi} \text { and } \\
\mathcal{S}_{0}=\frac{\xi \log _{e}^{2} \xi \log _{e} 2}{\theta T \alpha_{\mathrm{opt}}^{*}(1-\xi)\left(\frac{1}{T}\left(\sqrt{1+\frac{\gamma \bar{P} T}{N N_{0}}}-1\right)+\frac{\varphi \alpha_{\mathrm{opt}}^{*}}{2}\right)},
\end{gathered}
$$


respectively, where $\delta=\frac{\theta T \bar{P}}{N N_{0} \log _{e} 2}, \xi=1-e^{-\alpha_{\mathrm{opt}}^{*}}\left(1-e^{-\frac{\theta T \varphi \alpha_{\mathrm{opt}}^{*}}{\log _{e} 2}}\right)$, and $\varphi=\frac{\gamma \bar{P}}{N N_{0}}\left(\sqrt{1+\frac{N N_{0}}{\gamma P T}}-\sqrt{\frac{N N_{0}}{\gamma P T}}\right)^{2} \cdot \alpha_{\mathrm{opt}}^{*}$ is defined as $\alpha_{\mathrm{opt}}^{*}=\lim _{\zeta \rightarrow 0} \alpha_{\mathrm{opt}}$ and $\alpha_{\mathrm{opt}}^{*}$ satisfies

\section{Proof: See Appendix A.}

$$
\alpha_{\mathrm{opt}}^{*}=\frac{\log _{e} 2}{\theta T \varphi} \log _{e}\left(1+\frac{\theta T \varphi}{\log _{e} 2}\right) .
$$

Remark: We note that the minimum bit energy in the sparse multipath case with bounded degrees of freedom is achieved as $B \rightarrow \infty$ and hence as SNR $\rightarrow 0$. This is in stark contrast to the results in the low-power regime and rich multipath cases in which the bit energy requirements grow without bound as SNR vanishes. This is due to the fact that in sparse fading with bounded number of independent resolvable paths, uncertainty does not grow without bound because the number of subchannels $N$ is kept fixed as $B \rightarrow \infty$.

Remark: Theorem 4, through the minimum bit energy and wideband slope expressions, quantifies the bit energy requirements in the wideband regime when the system is operating subject to both statistical QoS constraints specified by $\theta$ and channel uncertainty. Note that both $\frac{E_{b}}{N_{0}}$ min and $\mathcal{S}_{0}$ depend on $\theta$ through $\delta$ and $\xi$. As will be observed in the numerical results, $\frac{E_{b}}{N_{0}}$ min and the bit energy requirements at nonzero spectral efficiency values generally increase with increasing $\theta$. Moreover, when compared with the results in Section IV, it will be seen that sparse multipath fading and having a bounded number of subchannels incur energy penalty no matter there is QoS constraints or not $(\theta=0)$, which is in stark contrast with previous results when there is perfect CSI at the receiver [22].

After having obtained analytical expressions for the minimum bit energy and wideband slope, we now provide numerical results. Fig. 7 plots the spectral efficiency-bit energy curve in the Rayleigh channel for different $\theta$ values. In the figure, we assume that $\bar{P} /\left(N N_{0}\right)=10^{4}$. As predicted, the minimum bit energies are obtained as SNR and hence the spectral efficiency approach zero. $\frac{E_{b}}{N_{0}}$ min are computed to be equal to $\{4.6776,4.7029,4.9177,6.3828,10.8333\} \mathrm{dB}$ for $\theta=\{0,0.001,0.01,0.1,1\}$, respectively. Moreover, the wideband slopes are $\mathcal{S}_{0}=\{0.4720,0.4749,0.4978,0.6151,0.6061\}$ for the same set of $\theta$ values. As can also be seen in the result of Theorem 4 , the minimum bit energy and wideband slope in general depend on $\theta$. In Fig. 7 , we note that the bit energy requirements (including the minimum bit energy) increase with increasing $\theta$, illustrating the energy costs of stringent queueing constraints. Finally, in this paper, we have considered fixed-rate/fixed-power transmissions over imperfectly-known channels. In Fig. 8, we compare the performance of this system with those in which the channel is perfectly-known and fixed- or variable-rate transmission is employed. The latter models have been studied in [21] and [22]. This figure demonstrates the energy costs of not knowing the channel and sending the information at fixed-rate. 
We finally consider the sparse multipath fading scenario (2b) in which the number of subchannels $N$ increases but only sublinearly with increasing bandwidth. Note that in this case, the bit energy required as $B \rightarrow \infty$ can be obtained by letting $N$ in the result of Theorem 4 , where $N$ is assumed to be fixed, go to infinity.

Corollary 1: In the wideband regime, if the number of subchannels $N$ increases sublinearly with $B$, then the bit energy required in the limit as $B \rightarrow \infty$ is

$$
\left.\frac{E_{b}}{N_{0}}\right|_{\mathrm{R}_{E}=0}=\infty
$$

Remark: As $N$ increases, each subchannel is allocated less power and operate in the low-power regime. Therefore, it is not surprising that we obtain the same bit energy result as in the low-power regime. Additionally, since the number of subchannels $N$ increases without bound, uncertainty in the channel increases as well. Hence, similarly as in rich multipath fading, extreme energy-inefficiency is experienced as $B \rightarrow \infty$.

Fig. 9 confirms the theoretical results. In this figure, we observe that the bit energy requirements initially decrease with decreasing spectral efficiency. However, below a certain spectral efficiency level, $\frac{E_{b}}{N_{0}}$ starts growing without bound for all $\theta \geq 0$.

\section{CONCLUSION}

In this paper, we have analyzed the energy efficiency of fixed-rate wireless transmissions for the communication scenario in which queueing constraints are present and the channel coefficients are estimated imperfectly by the receiver with the aid of training symbols. We have considered the effective capacity as a measure of the maximum throughput under statistical QoS constraints. We have identified the optimal fraction of power allocated to training and shown that this optimal fraction do not depend on the QoS exponent $\theta$ and the transmission rate. In particular, we have investigated the spectral efficiency-bit energy tradeoff in the low-power and wideband regimes. In the low-power regime, we have shown that the bit energy increases without bound as power diminishes. The minimum bit energy is achieved at a certain nonzero power level below which operation should be avoided. Although the minimum bit energy cannot be determined in closed-form, we have observed numerically that as QoS constraints become more stringent, the minimum bit energy increases. Similar results are obtained in the wideband regime as long as the number of subchannels increase without bound with increasing bandwidth as in rich multipath environments. On the other hand, if the number of subchannels remains bounded as the bandwidth increases, we have shown that 
the bit energy required at zero spectral efficiency (or equivalently at infinite bandwidth) is the minimum bit energy. We have noted that the minimum bit energy and wideband slope in general depend on the QoS exponent $\theta$. As the QoS constraints become more stringent and hence $\theta$ is increased, we have observed in the numerical results that the required minimum bit energy increases. Overall, we have quantified the increased energy requirements in the presence of QoS constraints in the low-power and wideband regimes, and identified the impact upon the energy efficiency of channel uncertainty and multipath sparsity and richness.

\section{APPENDIX}

\section{A. Proof of Theorem 4}

We first derive the following result for optimal fraction of power on training expressed in (21)

$$
\rho_{\mathrm{opt}}=\rho_{\mathrm{opt}}^{*}+\rho_{\mathrm{opt}}(0) \zeta+o(\zeta)
$$

where $\rho_{\mathrm{opt}}^{*}$ is a real value achieved as $\zeta \rightarrow 0$, and $\rho_{\mathrm{opt}}^{\cdot}(0)$ is the first derivative of $\rho_{\mathrm{opt}}$ evaluated at $\zeta=0$. We have

and

$$
\rho_{\mathrm{opt}}^{*}=\sqrt{\frac{N N_{0}}{\gamma \bar{P} T}\left(1+\frac{N N_{0}}{\gamma \bar{P} T}\right)}-\frac{N N_{0}}{\gamma \bar{P} T}
$$

$$
\rho_{\mathrm{opt}}(0)=\frac{1}{2 T} \sqrt{1+\frac{\gamma \bar{P} T}{N N_{0}}}\left(\sqrt{1+\frac{N N_{0}}{\gamma \bar{P} T}}-\sqrt{\frac{N N_{0}}{\gamma \bar{P} T}}\right)^{2} .
$$

Furthermore, $\mathrm{SNR}_{\text {eff,opt }}$ defined below equation (25) can be simplified to

$$
\mathrm{SNR}_{\mathrm{eff}, \mathrm{opt}}=\varphi \zeta+\omega \zeta^{2}+o\left(\zeta^{2}\right)
$$

where

$$
\varphi=\frac{\rho_{\mathrm{opt}}^{*}\left(1-\rho_{\mathrm{opt}}^{*}\right) \frac{\gamma^{2} \bar{P}^{2} T}{\left(N N_{0}\right)^{2}}}{1+\frac{\rho_{\mathrm{opt}}^{*} \bar{P} T}{N N_{0}}}=\frac{\gamma \bar{P}}{N N_{0}}\left(\sqrt{1+\frac{N N_{0}}{\gamma \bar{P} T}}-\sqrt{\frac{N N_{0}}{\gamma \bar{P} T}}\right)^{2}
$$

and

$$
\begin{aligned}
\omega & =\frac{\frac{\gamma^{2} P^{2} T}{N N_{0}^{2}}}{1+\rho_{\mathrm{opt}}^{*} \frac{\gamma \bar{P} T}{N N_{0}}}\left(\rho_{\mathrm{opt}}(0)\left(1-2 \rho_{\mathrm{opt}}^{*}\right)-\frac{\left(1-2 \rho_{\mathrm{opt}}^{*}\right) \frac{\gamma \bar{P}}{N N_{0}}+\rho_{\mathrm{opt}}^{\cdot}(0) \frac{\gamma \bar{P} T}{N N_{0}}-\frac{1}{T}}{1+\frac{\rho_{\mathrm{opt}}^{*} \gamma \bar{P} T}{N N_{0}}} \rho_{\mathrm{opt}}^{*}\left(1-\rho_{\mathrm{opt}}^{*}\right)\right) \\
& =-\frac{\gamma \bar{P}}{N N_{0} T}\left(\sqrt{1+\frac{N N_{0}}{\gamma \bar{P} T}}-\sqrt{\frac{N N_{0}}{\gamma \bar{P} T}}\right)^{2}\left(\sqrt{1+\frac{\gamma \bar{P} T}{N N_{0}}}-2\right) .
\end{aligned}
$$


Assume that the Taylor series expansion of $r_{\mathrm{opt}}$ with respect to small $\zeta$ is

$$
r_{\mathrm{opt}}=r_{\mathrm{opt}}^{*}+\dot{r}_{\mathrm{opt}}(0) \zeta+o(\zeta)
$$

where $r_{\mathrm{opt}}^{*}=\lim _{\zeta \rightarrow 0} r_{\mathrm{opt}}$ and $\dot{r}_{\mathrm{opt}}(0)$ is the first derivative with respect to $\zeta$ of $r_{\mathrm{opt}}$ evaluated at $\zeta=0$. From (10), we can find that

$$
\begin{aligned}
\alpha_{\mathrm{opt}} & =\frac{2^{\frac{r_{\mathrm{opt}} \zeta}{1-\zeta / T}}-1}{\mathrm{SNR}_{\mathrm{eff}, \mathrm{opt}}} \\
& =\frac{r_{\mathrm{opt}}^{*} \log _{e} 2+\left[\left(\frac{r_{\mathrm{opt}}^{*}}{T}+\dot{r}_{\mathrm{opt}}(0)\right) \log _{e} 2+\frac{\left(r_{\mathrm{opt}}^{*} \log _{e} 2\right)^{2}}{2}\right] \zeta+o(\zeta)}{\varphi+\omega \zeta+o(\zeta)} \\
& =\frac{r_{\mathrm{opt}}^{*} \log _{e} 2}{\varphi}+\left(\frac{\dot{r}_{\mathrm{opt}}(0) \log _{e} 2}{\varphi}+\frac{r_{\mathrm{opt}}^{*} \log _{e} 2}{\varphi}\left(\frac{1}{T}-\frac{\omega}{\varphi}\right)+\frac{\left(r_{\mathrm{opt}}^{*} \log _{e} 2\right)^{2}}{2 \varphi}\right) \zeta+o(\zeta)
\end{aligned}
$$

from which we have as $\zeta \rightarrow 0$ that

$$
\alpha_{\mathrm{opt}}^{*}=\frac{r_{\mathrm{opt}}^{*} \log _{e} 2}{\varphi}
$$

and that

$$
\dot{\alpha}_{\mathrm{opt}}(0)=\frac{\dot{r}_{\mathrm{opt}}(0) \log _{e} 2}{\varphi}+\frac{r_{\mathrm{opt}}^{*} \log _{e} 2}{\varphi}\left(\frac{1}{T}-\frac{\omega}{\varphi}\right)+\frac{\left(r_{\mathrm{opt}}^{*} \log _{e} 2\right)^{2}}{2 \varphi}
$$

where $\dot{\alpha}_{\mathrm{opt}}(0)$ is the first derivative with respect to $\zeta$ of $\alpha_{\mathrm{opt}}$ evaluated at $\zeta=0$. According to (52), $r_{\mathrm{opt}}^{*}=\frac{\varphi \alpha_{\mathrm{opt}}^{*}}{\log _{e} 2}$.

Combining with (46) and (52), we can obtain from (18) 4 as $\zeta \rightarrow 0$

$$
\frac{\log _{e} 2}{\varphi}\left(1-e^{-\frac{\theta T \varphi \alpha_{\mathrm{opt}}^{*}}{\log _{e} 2}}\right)-\theta T e^{-\theta T r_{\mathrm{opt}}^{*}}=0
$$

from which we get

$$
\alpha_{\mathrm{opt}}^{*}=\frac{\log _{e} 2}{\theta T \varphi} \log _{e}\left(1+\frac{\theta T \varphi}{\log _{e} 2}\right)
$$

Since $\frac{E_{b}}{N N_{0}}=\frac{\frac{\bar{P}}{N N_{0}}}{\frac{\mathrm{R}_{E}(\zeta)}{\zeta}}$, the result that $\left.\frac{E_{b}}{N N_{0}}\right|_{\mathrm{R}_{E}=0}=\frac{E_{b}}{N N_{0} \text { min }}$ follows from the fact that $\mathrm{R}_{E}(\zeta) / \zeta$ monotonically decreases with increasing $\zeta$, and hence achieves its maximum as $\zeta \rightarrow 0$. We now have

$$
\begin{aligned}
\frac{E_{b}}{N N_{0 \text { min }}} & =\lim _{\zeta \rightarrow 0} \frac{\frac{\bar{P}}{N N_{0}} \zeta}{\mathrm{R}_{E}(\zeta)}=\frac{-\frac{\theta T \bar{P}}{N N_{0}}}{\log _{e}\left(1-P\left\{|w|^{2} \geq \alpha_{\mathrm{opt}}^{*}\right\}\left(1-e^{\left.\left.-\theta T r_{\mathrm{opt}}^{*}\right)\right)}\right.\right.} \\
& =\frac{-\delta \log _{e} 2}{\log _{e} \xi}=\frac{\frac{\bar{P}}{N N_{0}}}{\dot{\mathrm{R}}_{E}(0)}
\end{aligned}
$$

where $\dot{\mathrm{R}}_{E}(0)$ is the derivative of $\mathrm{R}_{E}$ with respect to $\zeta$ at $\zeta=0, \delta=\frac{\theta T \bar{P}}{N N_{0} \log _{e} 2}$, and $\xi=1-P\left\{|w|^{2} \geq\right.$

${ }^{4} B$ is replaced by $B_{c}$ here according to 38 . 
$\left.\alpha_{\mathrm{opt}}^{*}\right\}\left(1-e^{-\frac{\theta T \varphi \alpha_{\mathrm{opt}}^{*}}{\log _{e} 2}}\right)$. Obviously, (57) provides (39).

Note that the second derivative $\ddot{\mathrm{R}}_{E}(0)$, required in the computation of the wideband slope $\mathcal{S}_{0}$, can be obtained from

$$
\begin{aligned}
\ddot{\mathrm{R}}_{E}(0) & =\lim _{\zeta \rightarrow 0} 2 \frac{\mathrm{R}_{E}(\zeta)-\dot{\mathrm{R}}_{E}(0) \zeta}{\zeta^{2}} \\
& =\lim _{\zeta \rightarrow 0} 2 \frac{1}{\zeta}\left(-\frac{1}{\theta T} \log _{e}\left(1-P\left\{|w|^{2} \geq \alpha_{\mathrm{opt}}\right\}\left(1-e^{-\theta T r_{\mathrm{opt}}}\right)\right)+\frac{1}{\theta T} \log _{e}\left(1-P\left\{|w|^{2} \geq \alpha_{\mathrm{opt}}^{*}\right\}\left(1-e^{-\theta T r_{\mathrm{opt}}^{*}}\right)\right)\right) \\
& =\lim _{\zeta \rightarrow 0}-\frac{2 e^{-\alpha_{\mathrm{opt}}}}{\theta T\left(1-P\left\{|w|^{2} \geq \alpha_{\mathrm{opt}}\right\}\left(1-e^{\left.\left.-\theta T r_{\mathrm{opt}}\right)\right)}\right.\right.}\left(\dot{\alpha}_{\mathrm{opt}}(\zeta)\left(1-e^{-\theta T r_{\mathrm{opt}}}\right)-\theta T e^{-\theta T r_{\mathrm{opt}}} \dot{\mathrm{opt}}_{\mathrm{opt}}(\zeta)\right) \\
& =-\frac{2 e^{-\alpha_{\mathrm{opt}}^{*}}}{\theta T\left(1-P\left\{|w|^{2} \geq \alpha_{\mathrm{opt}}^{*}\right\}\left(1-e^{\left.\left.-\theta T r_{\mathrm{opt}}^{*}\right)\right)}\right.\right.}\left(\dot{\alpha}_{\mathrm{opt}}(0)\left(1-e^{-\theta T r_{\mathrm{opt}}^{*}}\right)-\theta T e^{-\theta T r_{\mathrm{opt}}^{*}} \dot{r}_{\mathrm{opt}}(0)\right)
\end{aligned}
$$

where $r_{\mathrm{opt}}^{*}=\frac{\bar{P} \alpha_{\mathrm{opt}}^{*}}{N N_{0} \log _{e} 2}$. Above, (58) and (59) follow by using L'Hospital's Rule and applying Leibniz Integral Rule.

Meanwhile, substituting (54) and (53) into (59) gives us

$$
\begin{aligned}
\ddot{\mathrm{R}}_{E}(0) & =-\frac{2 e^{-\alpha_{\mathrm{opt}}^{*}}}{\theta T\left(1-P\left\{|w|^{2} \geq \alpha_{\mathrm{opt}}^{*}\right\}\left(1-e^{\left.-\theta T r_{\mathrm{opt}}^{*}\right)}\right.\right.} \alpha_{\mathrm{opt}}^{*}\left(1-e^{-\theta T r_{\mathrm{opt}}^{*}}\right)\left(\frac{1}{T}-\frac{\omega}{\varphi}+\frac{\varphi \alpha_{\mathrm{opt}}^{*}}{2}\right) \\
& =-\frac{2(1-\xi) \alpha_{\mathrm{opt}}^{*}}{\theta T \xi}\left(\frac{1}{T}-\frac{\omega}{\varphi}+\frac{\varphi \alpha_{\mathrm{opt}}^{*}}{2}\right) \\
& =-\frac{2(1-\xi) \alpha_{\mathrm{opt}}^{*}}{\theta T \xi}\left(\frac{1}{T}\left(\sqrt{1+\frac{\gamma \bar{P} T}{N N_{0}}}-1\right)+\frac{\varphi \alpha_{\mathrm{opt}}^{*}}{2}\right)
\end{aligned}
$$

Combining (60) and (57), we can prove (40).

\section{REFERENCES}

[1] B. Hassibi and B. M. Hochwald, "How much training is needed in multiple-antenna wireless links", IEEE Trans. Inform. Theory, vol. 49, pp. 951-963, Apr. 2003.

[2] M. C. Gursoy, "On the capacity and energy efficiency of training-based transmissions over fading channels," accepted for publication in the IEEE Trans. Inform. Theory, 2009. Available online at http://arxiv.org/abs/0712.3277.

[3] L. Tong, B. M. Sadler, and M. Dong, "Pilot-assisted wireless transmission," IEEE Signal Processing Mag., pp. 12-25, Nov. 2004.

[4] A. Lapidoth and S. Shamai (Shitz), "Fading channels: How perfect need 'perfect side information' be?," IEEE Trans. Inform. Theory, vol. 48, pp. 1118-1134, May 2002.

[5] D. Porrat, D. N. C. Tse, and S. Nacu, "Channel uncertainty in ultra-wideband communication systems," IEEE Trans. Inform. Theory, vol. 53, pp. 194-208, Jan. 2007.

[6] V. Raghavan, G. Hariharan, and A. M. Sayeed, "Capacity of sparse multipath channels in the ultra-wideband regime," IEEE Journ. Select. Topics in Signal Processing, vol. 1, pp. 357-371, Oct. 2007.

[7] E. Telatar and D. N. C. Tse, "Capacity and mutual information of wideband multipath fading channels," IEEE Trans. Inform. Theory, vol. 46, pp. 1384-1400, July 2000.

[8] S. Verdú, "Spectral efficiency in the wideband regime," IEEE Trans. Inform. Theory, vol.48, no.6 pp.1319-1343. Jun. 2002.

[9] A. Ephremides and B. Hajek, "Information theory and communication networks: An unconsummated union," IEEE Trans. Inform. Theory, vol. 44, pp. 2416-2434, Oct. 1998. 
[10] S. V. Hanly and D.N.C Tse, "Multiaccess fading channels-part II: delay-limited capacities," IEEE Trans. Inform. Theory, vol.44, no.7, pp.2816-2831. Nov. 1998.

[11] D. Wu and R. Negi "Effective capacity: a wireless link model for support of quality of service," IEEE Trans. Wireless Commun., vol.2,no. 4, pp.630-643. July 2003

[12] D. Wu and R. Negi,"Downlink scheduling in a cellular network for quality-of-service assurance, IEEE Trans. Veh. Technol., vol.53, no.5, pp. 1547-1557, Sep., 2004.

[13] D. Wu and R. Negi, "Utilizing multiuser diversity for efficient support of quality of service over a fading channel, IEEE Trans. Veh. Technol., vol.49, pp. 1073-1096, May 2003.

[14] J. Tang and X. Zhang, "Quality-of-service driven power and rate adaptation over wireless links, IEEE Trans. Wireless Commun., vol. 6, no. 8, pp.3058-3068, Aug. 2007.

[15] J. Tang and X. Zhang, "Quality-of-service driven power and rate adaptation for multichannel communications over wireless links, IEEE Trans. Wireless Commun., vol. 6, no. 12, pp.4349-4360, Dec. 2007.

[16] J. Tang and X. Zhang, "Cross-layer modeling for quality of service guarantees over wireless links, IEEE Trans. Wireless Commun., vol. 6, no. 12, pp.4504-4512, Dec. 2007.

[17] J. Tang and X. Zhang, "Cross-layer-model based adaptive resource allocation for statistical QoS guarantees in mobile wireless networks, IEEE Trans. Wireless Commun., vol. 7, pp.2318-2328, June 2008.

[18] L. Liu, P. Parag, J. Tang, W.-Y. Chen and J.-F. Chamberland, "Resource allocation and quality of service evaluation for wireless communication systems using fluid models," IEEE Trans. Inform. Theory, vol. 53, no. 5, pp. 1767-1777, May 2007

[19] L. Liu, P. Parag, and J.-F. Chamberland, "Quality of service analysis for wireless user-cooperation networks," IEEE Trans. Inform. Theory, vol. 53, no. 10, pp. 3833-3842, Oct. 2007

[20] L. Liu, and J.-F. Chamberland, "On the effective capacities of multiple-antenna Gaussian channels," IEEE International Symposium on Information Theory, Toronto, 2008.

[21] M.C. Gursoy, D. Qiao, and S. Velipasalar, "Analysis of energy efficiency in fading channel under QoS constrains," accepted to the IEEE Trans. Wireless Commun., 2009; conference version appeared at the IEEE Global Communications Conference (GLOBECOM), Dec. 2008.

[22] D. Qiao, M.C. Gursoy, and S. Velipasalar, "The impact of QoS constraints on the energy efficiency of fixed-rate wireless transmissions," submitted to the IEEE Trans. Wireless Commun., 2008; conference version to appear at the IEEE International Conference on Communications (ICC), Jun. 2009.

[23] C.-S. Chang, "Stability, queue length, and delay of deterministic and stochastic queuing networks," IEEE Trans. Auto. Control, vol. 39, no. 5, pp. 913-931, May 1994

[24] C.-S. Chang, Performance Guarantees in Communication Networks, New York: Springer, 1995

[25] C.-S. Chang and T. Zajic, "Effective bandwidths of departure processes from queues with time varying capacities," In Proceedings of IEEE Infocom, pp. 1001-1009, 1995

[26] P. Sadeghi and P. Rapajic, "Capacity analysis for finite-state Markov mapping of flat-fading channels," IEEE Trans. Commun. vol. 53, pp. 833-840, May 2005.

[27] A. Goldsmith, Wireless Communications, 1st ed. Cambridge University Press, 2005.

[28] Tim Szigeti and Christina Hattingh, End-to-End QoS Network Design: Quality of Service in LANs, WANs, and VPNs, Cisco Press, 2004.

[29] M. H. Protter, C. B. Morrey, and C. B. Morrey, Jr., A First Course in Real Analysis, 2nd ed., Springer, 1991.

[30] G. R. Grimmett and D. R. Stirzaker, Probability and Random Processes, 2nd ed. Oxford University Press, 1998.

[31] I. S. Gradshteyn and I. M. Ryzhik, Table of Integrals, Series, and Products, Academic Press, 2000. 


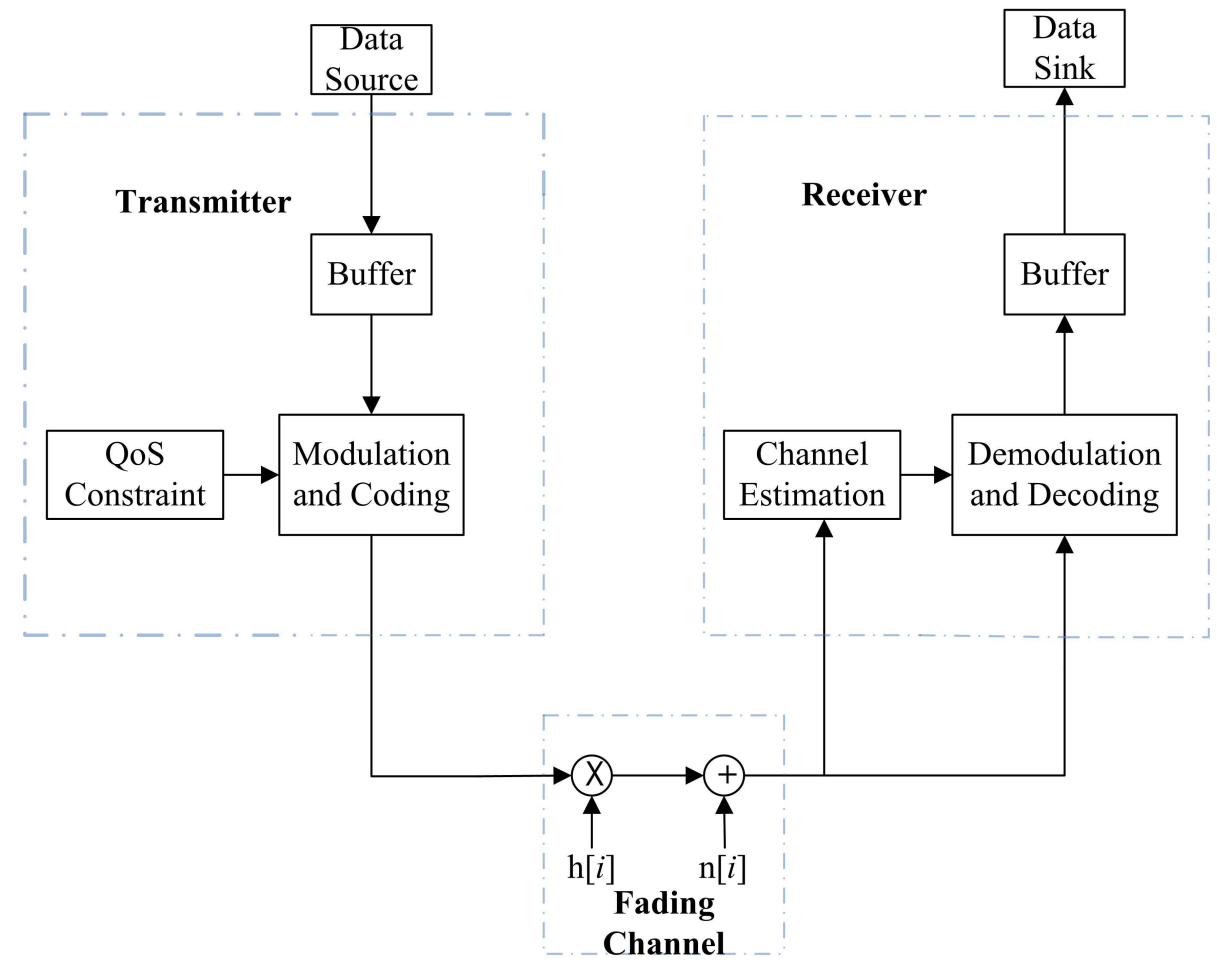

Fig. 1. The general system model.

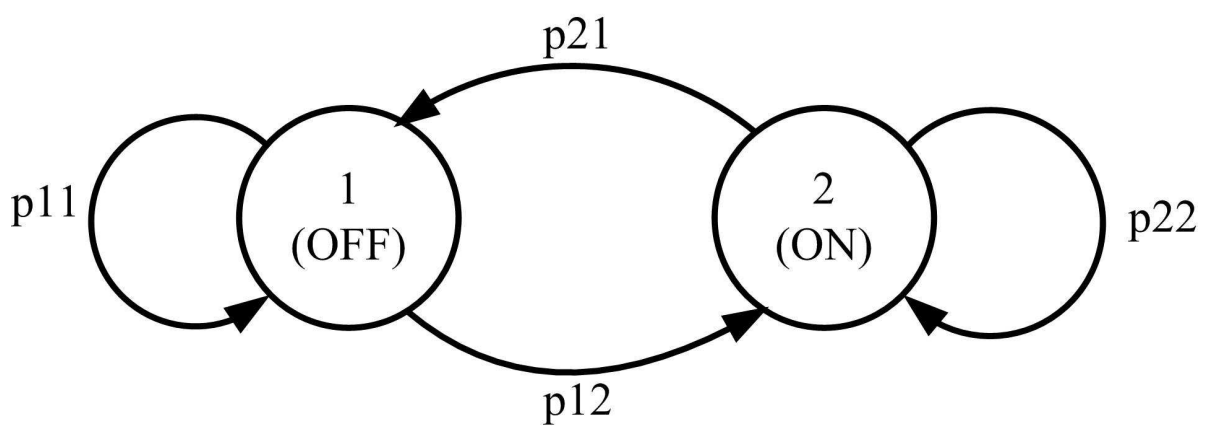

Fig. 2. ON-OFF state transition model. 


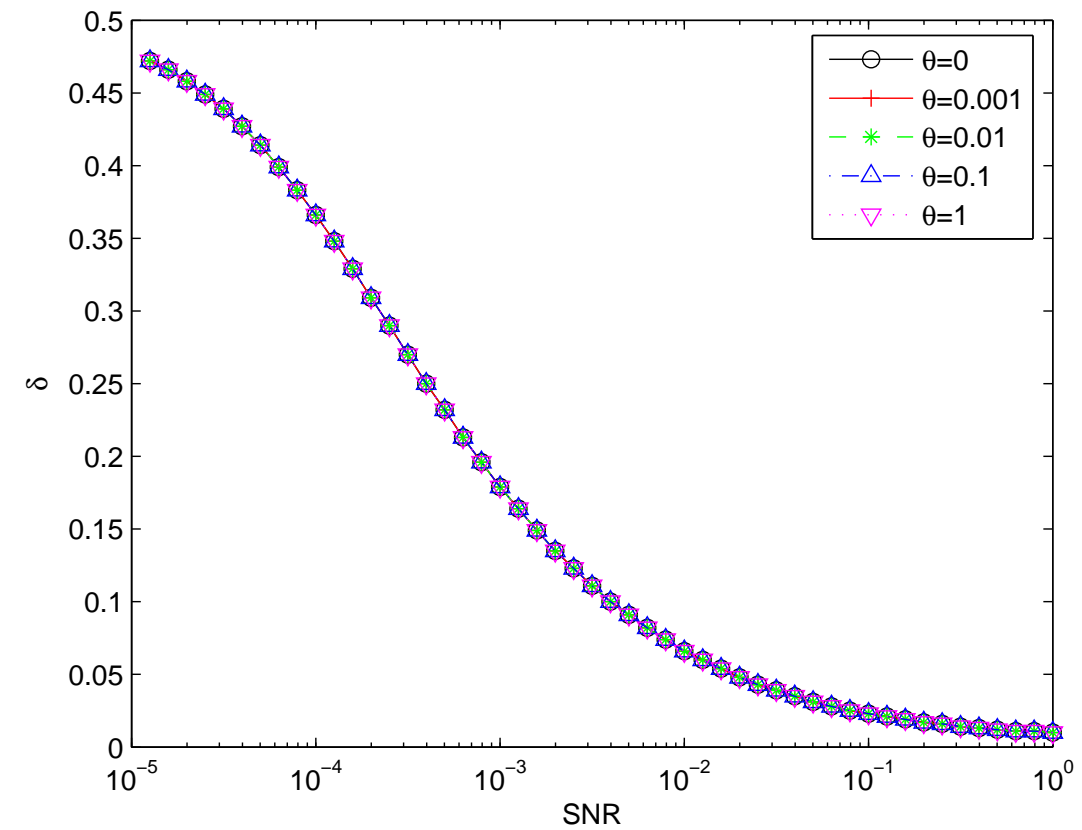

Fig. 3. Optimal fraction $\rho_{\text {opt }}$ vs. SNR in the Rayleigh channel. $B=10^{7} \mathrm{~Hz}$.

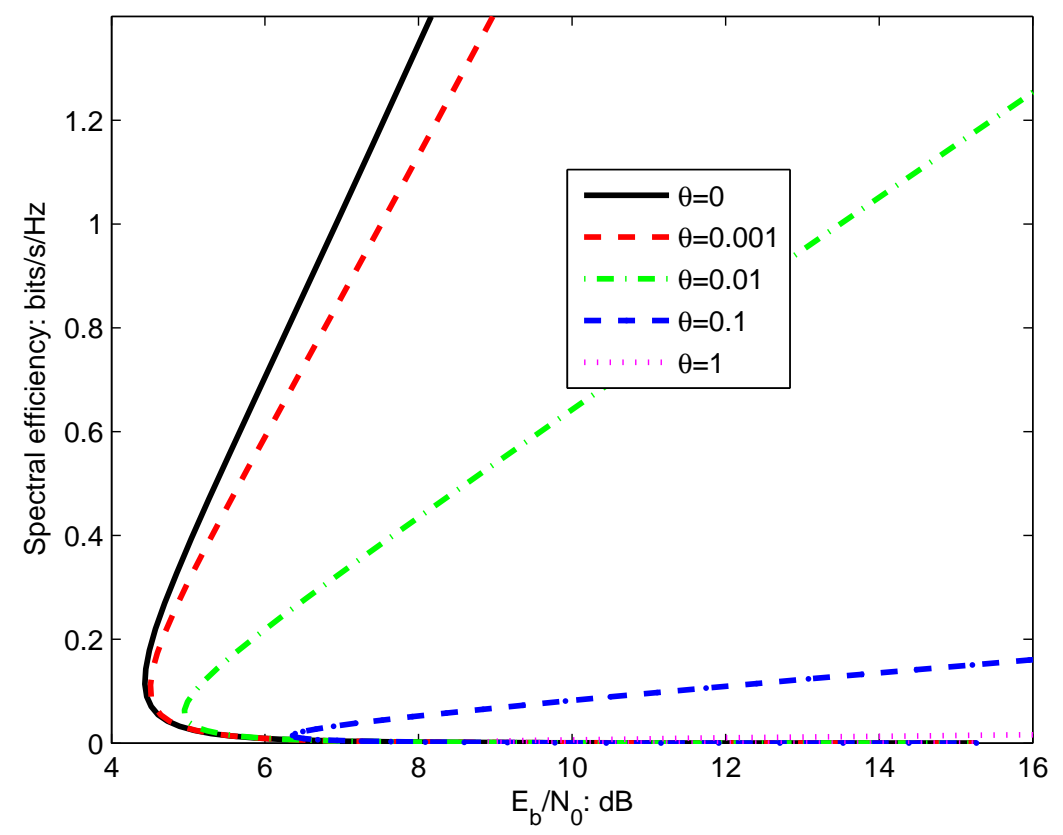

Fig. 4. Spectral efficiency vs. $E_{b} / N_{0}$ in the Rayleigh channel with $\mathbb{E}\left\{|h|^{2}\right\}=1$. $B=10^{5}$. 


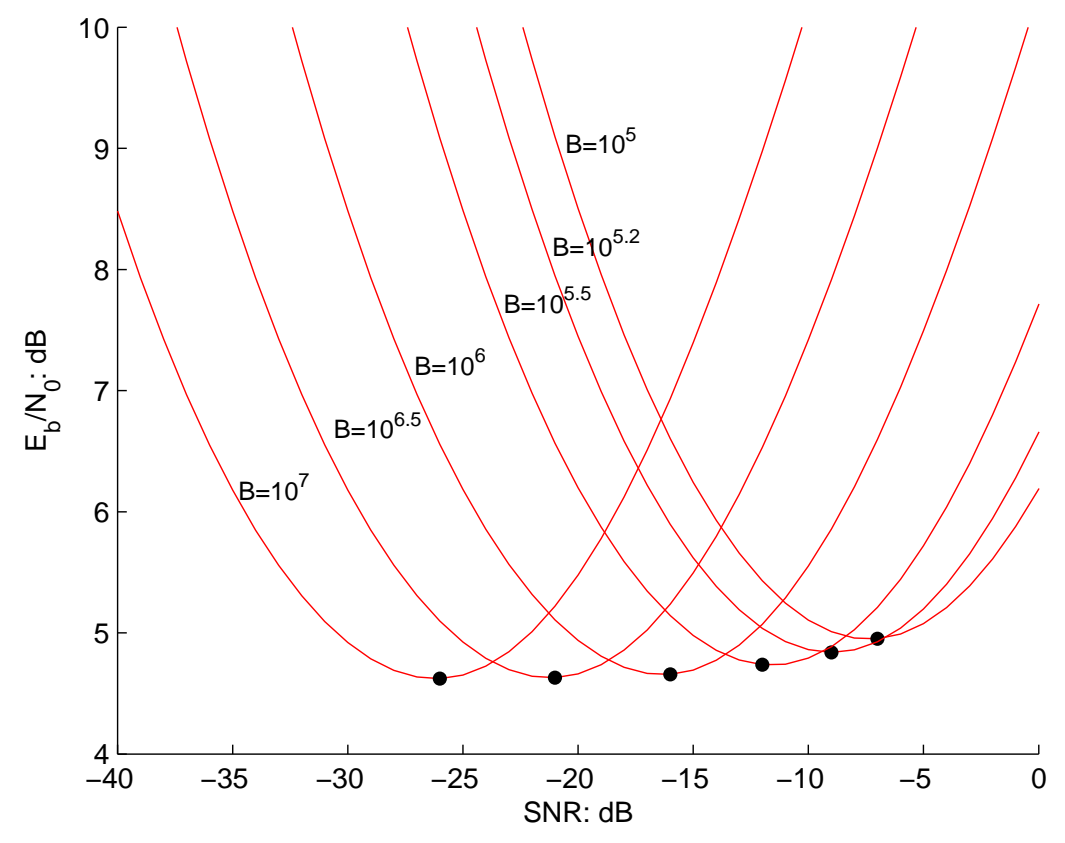

Fig. 5. $\quad E_{b} / N_{0}$ vs. SNR in the Rayleigh channel with $\mathbb{E}\left\{|h|^{2}\right\}=1 . \theta=0.01$.

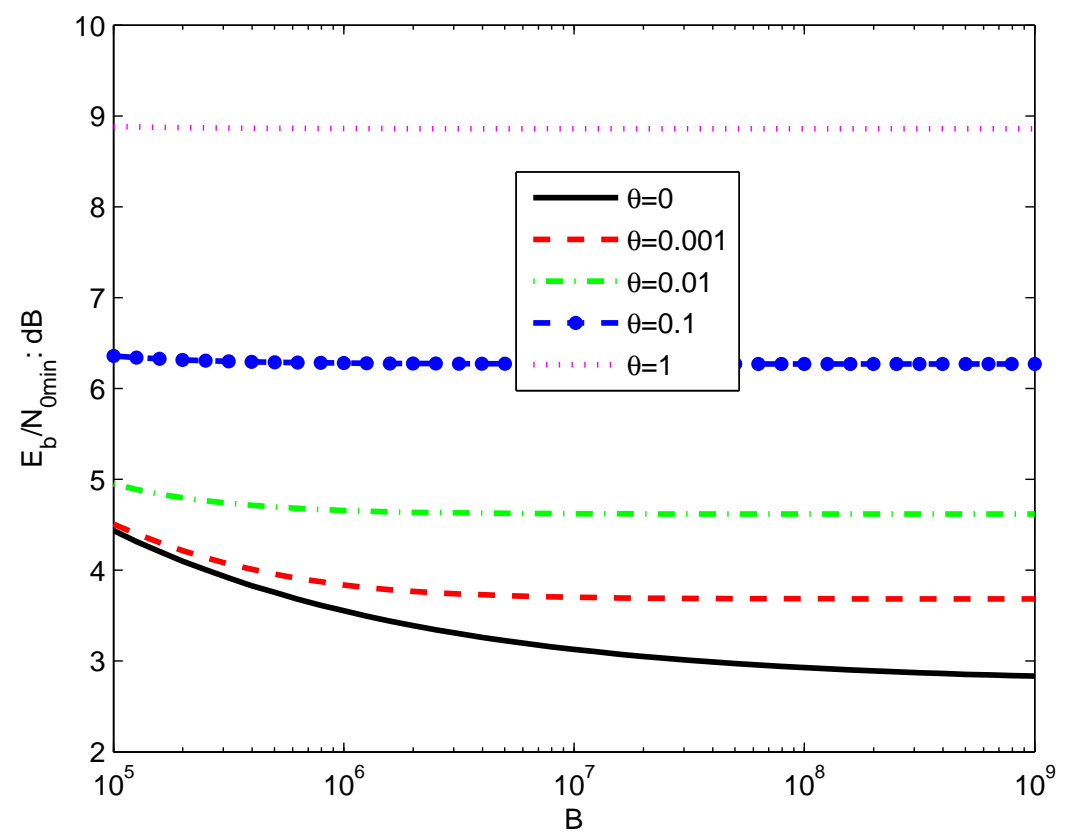

Fig. 6. $\frac{E_{b}}{N_{0}}$ min vs. $B$ in the Rayleigh channel with $\mathbb{E}\left\{|h|^{2}\right\}=1$. 


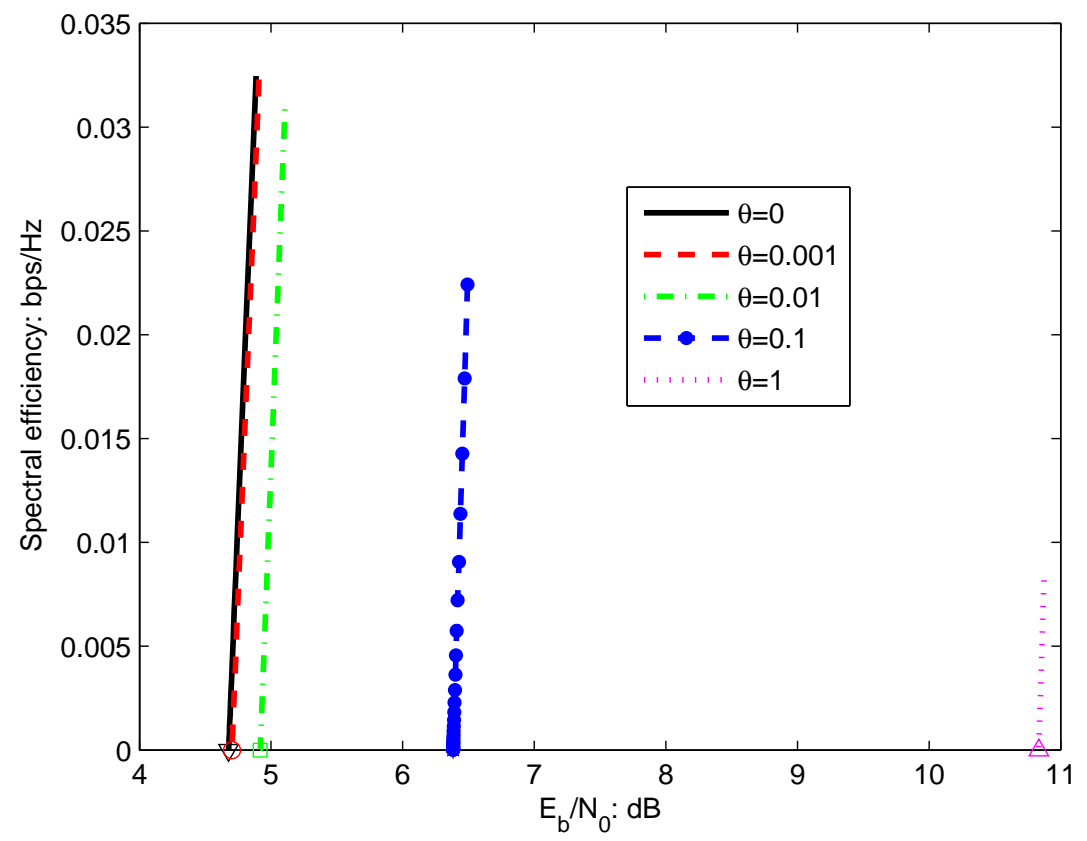

Fig. 7. Spectral efficiency vs. $E_{b} / N_{0}$ in the Rayleigh channel with $E\left\{|h|^{2}\right\}=\gamma=1 . \bar{P} / N N_{0}=10^{4}$.

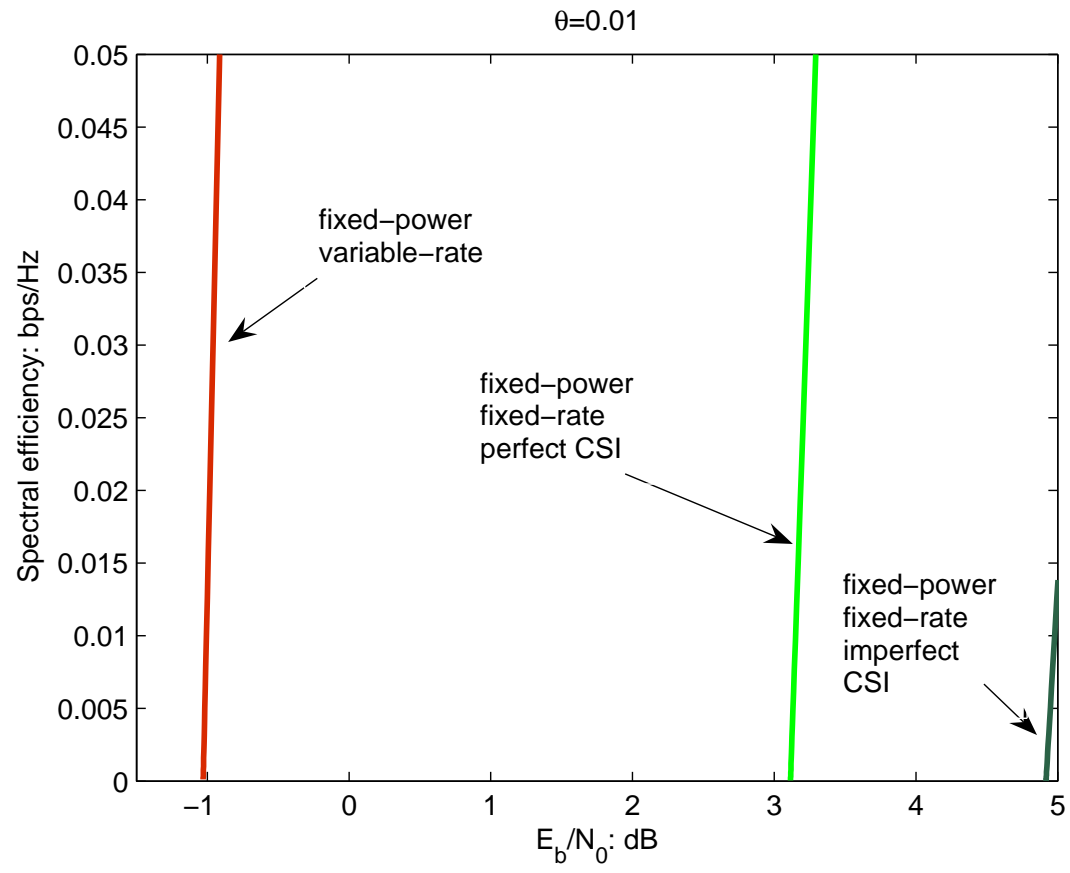

Fig. 8. Comparison of spectral efficiency; $\bar{P} / N N_{0}=10^{4}, \theta=0.01$, and $E\left\{|h|^{2}\right\}=\gamma=1$. 


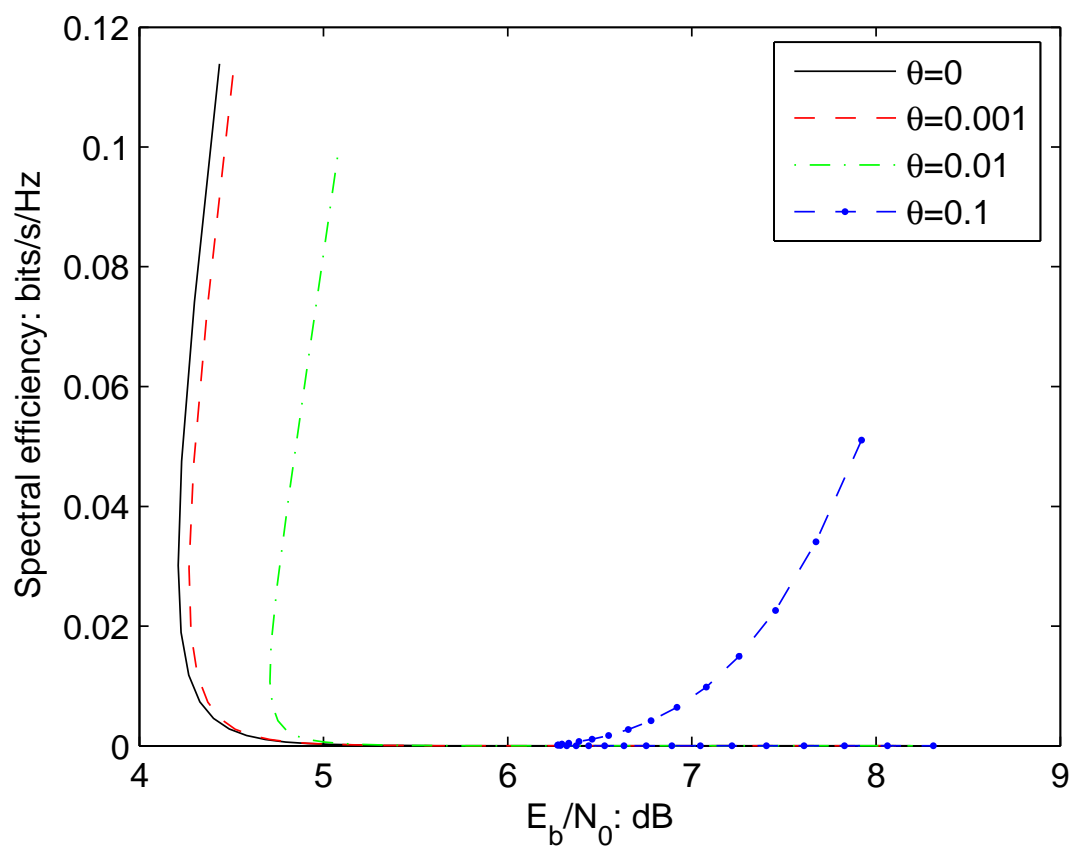

Fig. 9. Spectral efficiency vs. $E_{b} / N_{0}$ in the Rayleigh channel with $E\left\{|h|^{2}\right\}=\gamma=1 . \bar{P} / N N_{0}=10^{4}$. 